\title{
Depsipeptide cyclisation: Lactonization vs. lactamisation
}

\author{
Sacha Stadelmann, Stéphane Mayor, Roger Marti, Marc Mathieu
}

HESSO, Switzerland

https://doi.org/10.17952/35EPS.2018.166

The therapy of life-threatening infections significantly weakened by the global spread of antibiotic resistance has prompted an urgent need for the development of novel, effective, and safe antibiotics. Novel antibacterial agents with unprecedented mechanisms of action, which are devoid of pre-existing cross-resistances, are therefore necessary [1].

Peptides constitute one of the most promising platforms for drug development due to their biocompatibility, chemical diversity, and resemblance to proteins. Novel design approaches along with efficient and economic peptide synthesis have contributed to revitalize peptide-based drugs in the current pharmaceutical market [2]. Linear peptides are not very promising therapeutic agents owing to their low stability towards proteolysis, consequently reducing their feasibility and profitability for the pharmaceutical industry. Diverse chemical modification protocols that have evolved to diminish the mentioned drawbacks; these include cyclization, $\mathrm{N}$-methylation, incorporation of non-natural amino acids and other structural constraints.

Our literature searches brought us to consider natural products(actinomycetes), which have served as a promising source of new structural leads in the antibiotic resistance area and comply with these structural enhancing features (listed below), in particular the cyclodepsipeptides (CDP) [2]. CDP's show promising pharmacological activities and meet all criteria for good solubility and permeability. CDP's are capable like natural peptides, to interact with numerous proteins and show a large panel of activities (antitumor, anthelmintic, insecticidal, antibiotic, antifungal, immunosuppressant, anti-inflammatory and antimalarial).

However, further research of their medical applications is necessary and natural CDP's are challenging lead structures from a chemical and synthetic viewpoint [3], this triggered our attention. But, several StructureActivity Relationship (SAR) and medicinal chemistry studies enabled strong post-evolution of the natural scaffold and enabled us to design novel structures considering known issues. Limiting chemical factors described in the literature to include: (a) the lactone core can be hydrolyzed in basic and acidic aqueous media; (b) the acylated serine hydroxy group eliminated readily under non-aqueous basic conditions; (c) the conjugated triene was sensitive to temperature and light (cyclization and aromatization reactions) (defined in Figure 1 as the R group); (d) solubility was not sufficient for parenteral application.

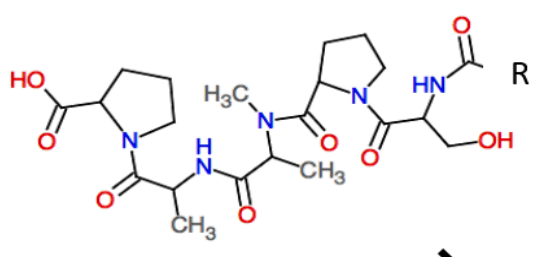

$\mathrm{R}$
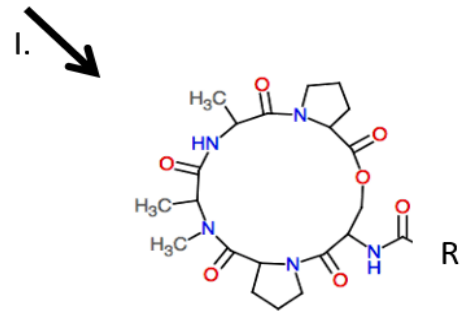<smiles>[R]C(=O)NC(COC(=O)C1CCCN1)C(=O)N1CCCC1C(=O)N(C)C(C)C(=O)NC(C)C(=O)O</smiles>

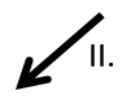

\section{$\mathrm{R}$}

Figure 1: Enopeptin A cyclisation strategies: (I) Lactonization vs. (II) Lactamisation. R=variable biocompatible functionality. 
Our solid-phase methodology contribution focuses on the evaluation of two distinct strategies for the cyclisation process preserving the ester functionality: (I) lactonization and (II) lactamisation are evaluated in order to produce Enopeptin A analogs $\left(\mathrm{R}=\right.$ variable functionality), as presented in the Figure 1. Lactonisation ${ }^{(\mathrm{I})}$ with our sequences did not give satisfying results, regardless of the chosen route: (i) 2 eq HATU; 2.5 eq DIEA; $1 \mathrm{~h}$ at $0^{\circ} \mathrm{C}$ followed by $24 \mathrm{~h}$ at $25^{\circ} \mathrm{C}$ and (ii) using Yamaguchi reagents [4] (data not shown).

Lactamisation $^{(\mathrm{II})}$ in opposition gave the target products, although in poor yield. Different concentrations were tested, in order to minimize polymerization but no visible improvement could be achieved. Other cyclisation methods for the lactam formation are currently under investigation.

\section{References}

1. Hinzen B. et al., Med. Chem., 2006, 1, pp. 689 - 693, DOI:

2. Tapeinou A. et al., Pep. Sci., 2015, 5, vol. 104,

3. Jordan D. Goodreid, J. Nat. Prod. 2014, 77, pp. 2170-2181 DOI: 10.1021/np500158q

4. Hikot M. et al., THL, 1990, 31, pp. 6367-6370 DOI: 